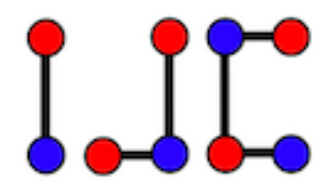

\title{
Some degree-based topological indices of triphenylene polyester
}

\author{
Muge Togan, Aysun Yurttas Gunes, Musa Demirci, Ismail Naci Cangul \\ Bursa Uludag University, Mathematics Department, Gorukle 16059 Bursa TURKEY \\ capkinm@uludag.edu.tr, ayurttas@uludag.edu.tr, mdemirci@uludag.edu.tr, cangul@uludag.edu.tr
}

\begin{abstract}
Molecules can be modelled by graphs to obtain their required properties by means of only mathematical methods and formulae. In this paper, several degree-based graph indices of one of the important chemical compounds called as polyester are calculated to determine several chemical and physiochemical properties of polyester.

Keywords: Polyester, graph, topological index

Mathematics Subject Classification: 05C07, 05C10, 05C30, 68R10

DOI: $10.19184 /$ ijc.2021.5.1.4
\end{abstract}

\section{Introduction}

Discotic liquid crystals (DLCs) are important nanostructures having applications in electronics and optoelectronics. Chandrasekhar et al. reported in 1977 that compounds in disc-like molecular shapes are also able to have mesophases [7]. Since then, efforts have been spent to molecular modification of triphenylene DLCs due to the advantages with their synthesis, chemically and thermally stablity and more importantly richness of mesophases.

Schultz isolated the symmetrical form of the fused aromatic hydrocarbon triphenylene known by the chemists for more than a century and named it triphenylene, see Fig. 1, [5]. The potential of triphenylene as an important source of DLCs was recognized in 1978, [3], after introduction of the notion of mesomorphism that the disc-shaped molecules have. Since that time, triphenylene has been in the focus of chemists especially researchers of liquid crystals as the derivatives

Received: 24 May 2020, Revised: 26 August 2020, Accepted: 10 February 2021. 


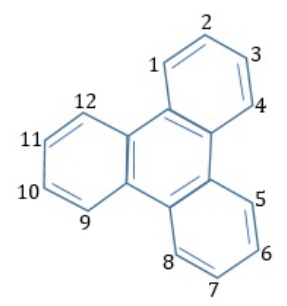

Figure 1. Triphenylene

of triphenylene are chemically and thermally stable and their chemistry is rather easy. They have several mesophases and their charge and energy transfer properties have the advantage of having the possibility of potential applications. Hence, some synthetic efforts has been put forward to obtain triphenylene-based DLCs [4, 6]. Such materials are studied due to their physical properties like one-dimensional energy transfer [15], one-dimensional charge transfer [2], [13], electroluminescence [18], ferroelectric switching [12], self-assembling behaviour and alignment on surfaces [19] and many others. At the moment, approximately 500 triphenylene-based DLCs are known.

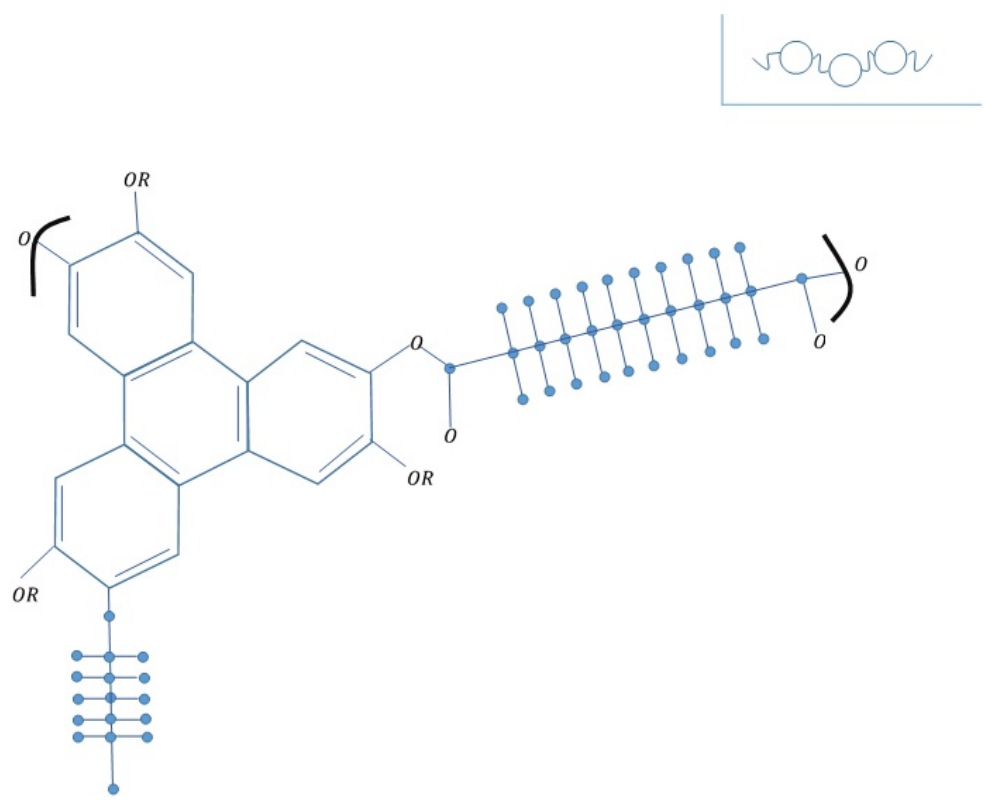

Figure 2. Triphenylene Polyester

Some molecular descriptors which are mathematical formulae called as graph indices have been defined and used since 1947 in many areas of science to study required properties of things such as molecules. Some graphs are produced from molecules by taking vertices for atoms and edges for chemical bonds. Indices are grouped into three families up to their definition: by vertex degrees, by distances or by matrices. 
We take $G$ as a simple graph with order $n$ and size $m$. The degree of a vertex $v \in V(G)$ is shown by $d_{G}(v)$.

First and second Zagreb indices are the most frequently known degree-based graph indices

$$
M_{1}(G)=\sum_{u \in V(G)} d_{G}^{2}(u) \text { and } M_{2}(G)=\sum_{u v \in E(G)} d_{G}(u) d_{G}(v) .
$$

defined in [11] for their QSAR and QSPR applications. The first Zagreb index were studied in [8]. These indices of some graph operations were calculated in [9].

The forgotten index denoted by $F(G)$ was defined by $F(G)=\sum_{u \in V(G)} d_{G}^{3}(u)$ and first appeared in 1972 [11] as an application of total $\pi$-electron energy calculation. It was indeed forgotten for some time and not further studied until a recent study [10].

A recently defined type of Zagreb indices is the hyper-Zagreb index defined by

$$
H M(G)=\sum_{(u v \in E}\left(d_{u}+d_{v}\right)^{2}
$$

see [10].

Heat of formation of octanes and heptanes inspired Furtula et al. to introduce the augmented Zagreb index having a better prediction and defined by

$$
A Z I(G)=\sum_{u v \in E(G)} \frac{d_{u} d_{v}}{d_{u}+d_{v}-2}
$$

The famous harmonic index was defined in [20] where it was correlated with П-electron energy of some benzenoid hydrocarbons:

$$
H(G)=\sum_{u v \in E(G)} \frac{2}{d_{u}+d_{v}}
$$

The redefined first, second and third Zagreb indices were respectively defined in [17] by

$$
\begin{gathered}
\operatorname{Re} Z G_{1}(G)=\sum_{u v \in E(G)} \frac{d_{u}+d_{v}}{d_{u} \cdot d_{v}}, \\
\operatorname{Re} Z G_{2}(G)=\sum_{u v \in E(G)} \frac{d_{u} \cdot d_{v}}{d_{u}+d_{v}}, \\
R e Z G_{3}(G)=\sum_{u v \in E(G)}\left(d_{u} \cdot d_{v}\right)\left(d_{u}+d_{v}\right) .
\end{gathered}
$$


Similarly, as an another variant, the reformulated Zagreb indices were defined in [16] by means of edge degrees as

$$
\begin{gathered}
R M_{1}(G)=\sum_{u v \in E(G)} d(e)^{2}, \\
R M_{2}(G)=\sum_{e, e^{\prime} \in E(G)} d(e) d\left(e^{\prime}\right)
\end{gathered}
$$

where $e, e^{\prime}$ are incident edge pairs of $G$.

In [1], reformulated $F$-index was defined by

$$
R F(G)=\sum_{u v \in E(G)} d(u v)^{3} .
$$

The first and second Banhatti indices were defined in [14] by taking into account pairs of all incident elements:

$$
\begin{gathered}
B_{1}(G)=\sum_{u, e}\left[d_{G}(u)+d(e)\right], \\
B_{2}(G)=\sum_{u, e} d_{G}(u) d(e) .
\end{gathered}
$$

\section{Main Results}

Now we will calculate the above topological indices of triphenylene polyester $P$. Our proofs depend on counting the edges according to their vertex degrees called as edge partition. First we obtain the partitions of the edge sets of the given graph and then we do the enumerative calculations. We start with two classical Zagreb indices:

Lemma 2.1. The two main Zagreb indices of polyester $P$ are $M_{1}(P)=720 \cdot n$ and $M_{2}(P)=$ $919 \cdot n$, respectively.

Proof. Let $u v$ be an edge of $P . E_{\left(d_{u}, d_{v}\right)}$ denotes the set of all edges with end vertex degrees $d_{u}$ and $d_{v}$. We partition the edges of $P$ according to their types. In $P$, we have the edges of type $E_{(1,3)}, E_{(1,4)}, E_{(2,3)}, E_{(2,4)}, E_{(3,3)}, E_{(3,4)}$ and $E_{(4,4)}$. The cardinalities of these sets in one unit are 2, 64, 20, 4, 9, 2 and 25, respectively. First, we calculate $M_{1}(P)$ for one unit:

$$
\begin{aligned}
M_{1}(P) & =\left|E_{(1,3)}\right|(1+3)+\left|E_{(1,4)}\right|(1+4)+\left|E_{(2,3)}\right|(2+3) \\
& +\left|E_{(2,4)}\right|(2+4)+\left|E_{(3,3)}\right|(3+3) \\
& +\left|E_{(3,4)}\right|(3+4)+\left|E_{(4,4)}\right|(4+4) \\
& =2 \cdot(1+3)+64 \cdot(1+4)+20 \cdot(2+3)+4 \cdot(2+4) \\
& +9 \cdot(3+3)+2 \cdot(3+4)+25 \cdot(4+4) \\
& =720
\end{aligned}
$$


and generalizing this to $n$ units, we get $M_{1}(P)=720 \cdot n$.

By the definition of $M_{2}(G)$, we can write

$$
\begin{aligned}
M_{2}(P) & =\left|E_{(1,3)}\right|(1 \cdot 3)+\left|E_{(1,4)}\right|(1 \cdot 4)+\left|E_{(2,3)}\right|(2 \cdot 3) \\
& +\left|E_{(2,4)}\right|(2 \cdot 4)+\left|E_{(3,3)}\right|(3 \cdot 3) \\
& +\left|E_{(3,4)}\right|(3 \cdot 4)+\left|E_{(4,4)}\right|(4 \cdot 4) \\
& =2 \cdot(1 \cdot 3)+64 \cdot(1 \cdot 4)+20 \cdot(2 \cdot 3)+4 \cdot(2 \cdot 4) \\
& +9 \cdot(3 \cdot 3)+2 \cdot(3 \cdot 4)+25 \cdot(4 \cdot 4) \\
& =919 .
\end{aligned}
$$

For $n$ units, we get $M_{2}(P)=919 \cdot n$.

Lemma 2.2. The third Zagreb index (forgotten index) of polyester $P$ is $F(P)=2460 \cdot n$.

Proof. We know that $F(G)=\sum_{u \in V(G)} d_{u}^{3}$, i.e.,

$$
\begin{aligned}
F(P) & =\sum_{u \in V} d_{u}{ }^{3}=1^{3} \cdot 66+2^{3} \cdot 12+3^{3} \cdot 14+4^{3} \cdot 30 \\
& =2460 .
\end{aligned}
$$

For $n$ units, we obtain $F(P)=2460 \cdot n$.

Lemma 2.3. The hyper-Zagreb index of polyester $P$ is $H M(P)=42984 \cdot n$.

Proof. We first have $H M(G)=\sum_{u v \in E(G)}\left(d_{u}+d_{v}\right)^{2}$, i.e.

$$
\begin{aligned}
H M(P) & =\left|E_{(1,3)}\right|(1+3)^{2}+\left|E_{(1,4)}\right|(1+4)^{2} \\
& +\left|E_{(2,3)}\right|(2+3)^{2}+\left|E_{(2,4)}\right|(2+4)^{2}+\left|E_{(3,3)}\right|(3+3)^{2} \\
& +\left|E_{(3,4)}\right|(3+4)^{2}+\left|E_{(4,4)}\right|(4+4)^{2} \\
& =2(1+3)^{2}+64(1+4)^{2}+20(2+3)^{2} \\
& +4(2+4)^{2}+9(3+3)^{2}+2(3+4)^{2}+25(4+4)^{2}=4298
\end{aligned}
$$

and for $n$ units, we get $H M(P)=4298 \cdot n$.

Lemma 2.4. The augmented Zagreb index of polyester $P$ is $A Z I(P)=954,692 \cdot n$. 
Proof. We know that $A Z I(G)=\sum_{u v \in E(G)}\left(\frac{d_{u} \cdot d_{v}}{d_{u}+d_{v}-2}\right)^{3}$, i.e.

$$
\begin{aligned}
A Z I(P) & =\left|E_{(1,3)}\right|\left(\frac{1 \cdot 3}{1+3-2}\right)^{3}+\left|E_{(1,4)}\right|\left(\frac{1 \cdot 4}{1+4-2}\right)^{3} \\
& +\left|E_{(2,3)}\right|\left(\frac{2 \cdot 3}{2+3-2}\right)^{3}+\left|E_{(2,4)}\right|\left(\frac{2 \cdot 4}{2+4-2}\right)^{3} \\
& +\left|E_{(3,3)}\right|\left(\frac{3 \cdot 3}{3+3-2}\right)^{3}+\left|E_{(3,4)}\right|\left(\frac{3 \cdot 4}{3+4-2}\right)^{3} \\
& +\left|E_{(4,4)}\right|\left(\frac{4 \cdot 4}{4+4-2}\right)^{3} \\
& =2\left(\frac{3}{2}\right)^{3}+64\left(\frac{4}{3}\right)^{3}+160+32+9\left(\frac{9}{4}\right)^{3}+2\left(\frac{12}{5}\right)^{3}+25\left(\frac{8}{3}\right)^{3} \\
& =954,692
\end{aligned}
$$

giving the result.

Lemma 2.5. The harmonic index of $P$ is $H(P)=45,754 \cdot n$.

Proof. Recall that $H(G)=\sum_{u v \in E(G)} \frac{2}{d_{u}+d_{v}}$, i.e.,

$$
\begin{aligned}
H(P) & =\left|E_{(1,3)}\right| \frac{2}{1+3}+\left|E_{(1,4)}\right| \frac{2}{1+4}+\left|E_{(2,3)}\right| \frac{2}{2+3} \\
& +\left|E_{(2,4)}\right| \frac{2}{2+4}+\left|E_{(3,3)}\right| \frac{2}{3+3}+\left|E_{(3,4)}\right| \frac{2}{3+4}+\left|E_{(4,4)}\right| \frac{2}{4+4} \\
& =2 \cdot \frac{1}{2}+64 \cdot \frac{2}{5}+20 \cdot \frac{2}{5}+4 \cdot \frac{1}{3}+9 \cdot \frac{1}{3}+2 \cdot \frac{2}{7}+25 \cdot \frac{1}{4}=45,754
\end{aligned}
$$

and for $n$ units, we get $H(P)=45,754 \cdot n$.

Lemma 2.6. The re-defined Zagreb indices of $P$ are $\operatorname{Re} Z G_{1}(P)=122,01 \cdot n$, $\operatorname{Re} Z G_{2}(P)=$ $148,96 \cdot n$ and $\operatorname{Re} Z G_{3}(P)=5950 \cdot n$.

Proof. Recall that $\operatorname{Re} Z G_{1}(G)=\sum_{u v \in E(G)} \frac{d_{u}+d_{v}}{d_{u} \cdot d_{v}}$, i.e.,

$$
\begin{aligned}
\operatorname{Re} Z G_{1}(P) & =\left|E_{(1,3)}\right| \frac{1+3}{1 \cdot 3}+\left|E_{(1,4)}\right| \frac{1+4}{1 \cdot 4}+\left|E_{(2,3)}\right| \frac{2+3}{2 \cdot 3} \\
& +\left|E_{(2,4)}\right| \frac{2+4}{2 \cdot 4}+\left|E_{(3,3)}\right| \frac{3+3}{3 \cdot 3}++\left|E_{(3,4)}\right| \frac{3+4}{3 \cdot 4} \\
& +\left|E_{(4,4)}\right| \frac{4+4}{4 \cdot 4} \\
& =2 \cdot \frac{4}{3}+64 \cdot \frac{5}{4}+20 \cdot \frac{5}{6}+4 \cdot \frac{3}{4}+9 \cdot \frac{2}{3}+2 \cdot \frac{7}{12}+25 \cdot \frac{1}{2}=122,01 .
\end{aligned}
$$

For $n$ units, we get the result $\operatorname{Re} Z G_{1}(P)=122,01 \cdot n$.

As $\operatorname{Re} Z G_{2}(G)=\sum_{u v \in E(G)} \frac{d_{u} \cdot d_{v}}{d_{u}+d_{v}}$ and $\operatorname{Re} Z G_{3}(G)=\sum_{u v \in E(G)}\left(d_{u} \cdot d_{v}\right)\left(d_{u}+d_{v}\right)$, by using the similar methods we get the results for $\operatorname{Re} Z G_{2}(P)$ and $\operatorname{Re} Z G_{3}(P)$. 
Lemma 2.7. The reformulated Zagreb indices of $P$ are $R M_{1}(P)=1922 \cdot n$ and $R M_{2}(P)=$ $3859 \cdot n$, respectively.

Proof. In $P$, the degrees of the edges $d(u v)$ where $u v$ is an edge are $2,3,4,5$ and 6 . The number of these edge degrees of $P$ are $|d(u v)=2|=2,|d(u v)=3|=84,|d(u v)=4|=13, \mid d(u v)=$ $5 \mid=2$ and $|d(u v)=6|=25$, respectively. Hence

$$
R M_{1}(P)=2 \cdot 2^{2}+84 \cdot 3^{2}+13 \cdot 4^{2}+2 \cdot 5^{2}+25 \cdot 6^{2}=1922 .
$$

For $n$ units, we find $R M_{1}(P)=1922 \cdot n$.

For calculating $R M_{2}(P)$, we partition the incident edges of $P$ according to product of their edge degrees $d(e) \cdot d\left(e^{\prime}\right)$ where $e, e^{\prime} \in E$ and $e e^{\prime}$. In $P$, we get $d(2) \cdot d(3), d(2) \cdot d(5), d(3) \cdot$ $d(3), d(3) \cdot d(4), d(3) \cdot d(5), d(3) \cdot d(6), d(4) \cdot d(4), d(4) \cdot d(6), d(5) \cdot d(6)$ and $d(6) \cdot d(6)$. The number of these type of products are 2, 2, 53, 32, 6, 104, 8, 4, 2 and 20, respectively. Hence

$$
\begin{aligned}
R M_{2}(P) & =|d(2) \cdot d(3)| \cdot 2 \cdot 3+|d(2) \cdot d(5)| \cdot 2 \cdot 5 \\
& +|d(3) \cdot d(3)| \cdot 3 \cdot 3+|d(3) \cdot d(4)| \cdot 3 \cdot 4+|d(3) \cdot d(5)| \cdot 3 \cdot 5 \\
& +|d(3) \cdot d(6)| \cdot 3 \cdot 6+|d(4) \cdot d(4)| \cdot 4 \cdot 4+|d(4) \cdot d(6)| \cdot 4 \cdot 6 \\
& +|d(5) \cdot d(6)| \cdot 5 \cdot 6+|d(6) \cdot d(6)| \cdot 6 \cdot 6 \\
& =2 \cdot 6+2 \cdot 10+53 \cdot 9+32 \cdot 12+6 \cdot 15+104 \cdot 18+8 \cdot 16+4 \cdot 24 \\
& +2 \cdot 30+20 \cdot 36 \\
& =3859 .
\end{aligned}
$$

For $n$ units, $R M_{2}(P)=3859 \cdot n$.

Lemma 2.8. The reformulated $F$-index of $P$ are $R F(P)=8766 \cdot n$, respectively.

Proof. Recall that $R F(G)=\sum_{u v \in E(G)} d(u v)^{3}$, i.e.,

$$
\begin{aligned}
R F(P) & =|d(u v)=2| \cdot 2^{3}+|d(u v)=3| \cdot 3^{3}+|d(u v)=4| \cdot 4^{3}+|d(u v)=5| \cdot 5^{3} \\
& +|d(u v)=6| \cdot 6^{3} \\
& =2 \cdot 2^{3}+84 \cdot 3^{3}+13 \cdot 4^{3}+2 \cdot 5^{3}+25 \cdot 6^{3}=8766 .
\end{aligned}
$$

For $n$ units, we get $R F(P)=8766 \cdot n$.

Lemma 2.9. The Banhatti indices of $P$ are $B_{1}(P)=1656 \cdot n$ and $B_{2}(P)=2854 \cdot n$, respectively.

Proof. We know that $B_{1}(G)=\sum_{u, e} d_{G}(u)+d(e)$, i.e.,

$$
\begin{aligned}
B_{1}(P) & =2[(1+2)+(2+3)]+64[(4+3)+(1+3)]+20[(2+3)+(3+3)] \\
& +9[(3+4)+(3+4)]+4[(4+4)+(2+4)]+2[(4+5)+(3+5)] \\
& +25[(6+4)+(6+4)] \\
& =1656
\end{aligned}
$$


and for $n$ units, $B_{1}(P)=1656 \cdot n$.

$$
\begin{aligned}
& \text { Recall that } B_{2}(G)=\sum_{u, e} d_{G}(u) \cdot d(e) \text {, i.e. } \\
& \qquad \begin{aligned}
B_{2}(P) & =2[(1 \cdot 2)+(2 \cdot 3)]+64[(4 \cdot 3)+(1 \cdot 3)]+20[(2 \cdot 3)+(3 \cdot 3)] \\
+ & +9[(3 \cdot 4)+(3 \cdot 4)]+4[(4 \cdot 4)+(2 \cdot 4)]+2[(4 \cdot 5)+(3 \cdot 5)] \\
+ & 25[(6 \cdot 4)+(6 \cdot 4)] \\
& =2854
\end{aligned}
\end{aligned}
$$

and for $n$ units, $B_{2}(P)=2854 \cdot n$.

\section{Conclusion}

In the last seven decades, some mathematical descriptors have been defined and used by mathematicians and chemists to determine some physico chemical properties of certain molecules by mathematical calculations. In this paper, several such descriptors are calculated for triphenylene polyester shown in Fig. 2.

\section{References}

[1] H. Aram and N. Dehgardi, Reformulated F-index of graph operations, Commun. Comb. Optim. 2 (2017), 87-98.

[2] A. Bayer, S. Zimmermann and J. H. Wendorff, Mol. Cryst. Liq. Cryst. 396 (2003), 1.

[3] J. Billard, J. C. Dubois, N. H. Tinh and A. Zann, Nouv. J. Chimie 2 1978, 535.

[4] N. Boden, R. J. Bushby and A. N. Cammidge, J. Am. Chem. Soc. 117 (1995), 924.

[5] C. M. Buess and D. D. Lawson, Chem. Rev. 60 (1960), 313.

[6] A. N. Cammidge and R. J. Bushby, Handbook of Liquid Crystals, Vol.2B, edited by D. Demus, J. Goodby, G. W. Gray, H. W. Spiess and V. Vill, Wiley-VCH, Chap. VII, 1998.

[7] S. Chandrasekhar, B. K. Sadashiva and K. A. Suresh, Liquid crystals of disc-like molecules, Pramana 9 (1977), 471-480.

[8] K. C. Das, N. Akgunes, M. Togan, A. Yurttas, I. N. Cangul and A. S. Cevik, On the first Zagreb index and multiplicative Zagreb coindices of graphs, Analele Stiintifice ale Universitatii Ovidius Constanta 24 (1) (2016), 153-176.

[9] K. C. Das, A. Yurttas, M. Togan, I. N. Cangul and A. S. Cevik, The multiplicative Zagreb indices of graph operations, Journal of Inequalities and Applications 90 (2013).

[10] B. Furtula and I. Gutman, A Forgotten Topological Index, J. Math. Chem. 53 (4) (2015), 1184-1190. 
[11] I. Gutman and N. Trinajstic, Graph theory and molecular orbitals III. Total $\pi$-electron energy of alternant hydrocarbons, Chem. Phys. Lett. 17 (1972), 535-538.

[12] G. Heppke, D. Kruerke, C. Lohning, D. Lotzsch, D. Moro, M. Muller and H. Sawade, J. Mater. Chem. 10 (2000) 2657.

[13] E. I. Kats, Mol. Cryst. Liq. Cryst. 396 (2003), 23.

[14] V. R. Kulli, On K Banhatti indices of graphs, J. Comput. Math. Sci. 7 (2016), 213-218.

[15] S. Marguet, D. Markovitsi, P. Millie, H. Sigal and S. Kumar, J. Phys. Chem. 102 (1998), 4697.

[16] A. Milićević, S. Nikolić and N. Trinajstić, On reformulated Zagreb indices, Mol. Divers. 8 (2004), 393-399.

[17] P. S. Ranjini, V. Lokesha and A. Usha, Relation between phenylene and hexagonal squeeze using harmonic index. Int J Graph Theory 1 (2013), 116-121.

[18] I. Seguy, P. Destruel and H. Bock, Synth. Met. 15 (2000), 111-112.

[19] S. E. Tsvetkov, T. S. Perova, J. K. Vij, D. Simpson, S. Kumar and F. Vladimirov, Mol. Cryst. Liq. Cryst. 11 (1999), 267.

[20] L. Zhong, The harmonic index on graphs, Applied Mathematics Letters 25 (2012), 561-566. 